\title{
Effects and reproducibility of aerobic and resistance exercise on appetite and energy intake in young, physically active adults
}

\author{
Derek J. Laan, \\ Department of Foods and Nutrition, Purdue University, 700 West State Street, IN 47907, West \\ Lafayette, USA \\ Heather J. Leidy, \\ Department of Nutrition \& Exercise Physiology, University of Missouri, 204 Gywnn Hall, MO \\ 65201, Columbia, USA \\ Eunjung Lim, and \\ Department of Statistics, Purdue University, 250 North University Street, IN 47907, West \\ Lafayette, USA \\ Wayne W. Campbell ${ }^{1}$ \\ Department of Foods and Nutrition, Purdue University, 700 West State Street, IN 47907, West \\ Lafayette, USA
}

\begin{abstract}
Appetite and meal energy intake (MEI) following aerobic (AEx) and resistance (REx) exercises were evaluated in 19 young, active adults. The participants completed duplicate 35-min sessions of AEx, REx, and sedentary control, and consumed an ad libitum pasta meal 30 min postsession. Hunger transiently decreased after AEx but was not influenced by REx. MEI was $14 \%$ to $18 \%$ higher after AEx and REx than control. These findings are consistent with exercise-stimulated ingestive behavior, not anorexia of exercise.
\end{abstract}

\section{Keywords}

anorexia of exercise; aerobic exercise; appetite; dietary compensation; energy intake; hunger; strength exercise

\section{Introduction}

The term "anorexia of exercise" refers to the reduction of perceived hunger that may occur within 60 min following a bout of exercise at an intensity $>60 \%$ of maximum oxygen consumption $\left(V \dot{\mathrm{O}}_{2 \text { max }}\right)$ that is possibly related to the redistribution of blood flow from visceral organs to muscles (Blundell et al. 2003). The vast majority of research surrounding this topic has focused on higher intensity aerobic exercise and consistently illustrates declines in perceived hunger immediately following exercise (Thompson et al. 1988; Verger et al. 1992; Imbeault et al. 1997; Westerterp-Plantenga et al. 1997; Lluch et al. 2000;

\footnotetext{
${ }^{1}$ Corresponding author (campbellw@ purdue.edu).
} 
Pomerleau et al. 2004; Broom et al. 2009). Despite the documented reductions in appetite, conflicting evidence exists surrounding the changes in meal energy intake (MEI), with decreased (Verger et al. 1992; Westerterp-Plantenga et al. 1997), unchanged (Thompson et al. 1988; Imbeault et al. 1997; Lluch et al. 2000), and increased intake (Pomerleau et al. 2004) reported. The effect of resistance exercise (REx) on appetite and energy intake is also uncertain. According to Broom et al. (2009), REx suppresses hunger for up to $1 \mathrm{~h}$ compared with no exercise, but this response is less robust than a response to aerobic exercise (AEx). In contrast, Ballard et al. (2009) found no effect of REx on perceived hunger. Neither study evaluated postexercise energy intake, thus the effect of REx on MEI requires investigation. The primary objective of this study was to evaluate appetite sensations and MEI responses following REx, as compared with those of AEx or no-exercise control. We hypothesized that REx would not result in a short-term anorexia of exercise response, in contrast to aerobic exercise.

Previous studies where subjects were presented with a test meal (typically either a buffetstyle offering of food or a fixed macronutrient meal) on duplicate or triplicate days indicate that reproducibility is good (intra-class correlation, ICC $\searrow 0.80$ ) (Arvaniti et al. 2000; Choi et al. 2000; Gregersen et al. 2008; Nair et al. 2009). These studies were accomplished with the subjects in sedentary states, and the reproducibility of MEI postexercise is undocumented. In this study, the appetite sensations and energy intake responses to AEx, REx, and no-exercise control were evaluated twice in each subject. These duplicate trials provided a novel opportunity to assess the reproducibility of ad libitum MEI postexercise, which is a secondary objective of this study.

\section{Materials and methods}

\section{Subjects}

Men and women from the Purdue University and West Lafayette, Indiana, community were recruited using campus and community postings. Eligibility was based on the following criteria: (i) aged 18 to 29 years; (ii) body mass index (BMI) between 18 and $29 \mathrm{~kg} \cdot \mathrm{m}^{-2}$; (iii) percent body fat was $<20 \%$ for men and $<35 \%$ for women; (iv) not dieting and no weight loss or gain (>4.5 kg) within the past 6 months; (v) nonsmoking; (vi) nondiabetic; (vii) physically active (defined as participating in at least 2 AEx and 2 REx sessions of at least 30 min each per week for the last 3 months); and (viii) acceptance of the study food (pasta salad). All subjects were approved by the study physician and gave written informed consent. Twenty-nine study-eligible people agreed to participate and signed consent forms. Eight of these people did not start or complete the baseline testing because of unexpected lack of interest or commitment to fill out 3-day food records. One subject withdrew after completing 1 of the 6 treatment testing days because of a fractured hand unrelated to the study protocol, and 1 subject was excused after completing 1 treatment testing day because of scheduling and compliance issues. Nineteen participants (10 women, 9 men) completed all study procedures. The study was approved by the Purdue University Institutional Review Board, and the subjects received monetary compensation for participating. The ClinicalTrials.gov identifier is NCT00761163 (available from clinicaltrials.gov). 


\section{Baseline assessments}

Maximal strength, aerobic fitness, and body composition were measured at the start of the study. Percent body fat was estimated from body volume and mass using a plethysmograph with direct measurement of thoracic lung volume (BOD-POD, Life Measurement Instruments Inc., Concord, Calif.) and the Siri 2-compartment model equation (Siri 1956). Height was measured using a wall-mounted stadiometer. Maximum strength (i.e., 1 repetition maximum (1RM)) for chest press, double leg press, seated row, leg extension, and seated leg curl exercises was assessed using pneumatic resistance exercise equipment (Keiser Sports Health Equipment Company, Fresno, Calif.). Whole-body strength is the sum of the five 1RMs. The YMCA sub maximal cycle ergometry protocol was used to estimate each subject's $V \dot{O}_{2 \max }$ (ACSM 2006). A Monark Cycle Ergometer (Ergomedic 828E, Monark Exercise AB, Vansbro, Sweden) and Polar heart rate monitor (A1, Polar Electro, Oy, Finland) were used to perform the tests.

\section{Experimental design and procedures}

This randomized cross-over study incorporated a within-subject design with 3 treatments: AEx, 35 min of cycling at 70\% heart rate reserve; REx, 35 min of 3 sets at $70 \% 1 \mathrm{RM}$ for 5 exercises; and no exercise (i.e., control) (CON). The randomization was done by co-author H.J. Leidy using a table of random numbers (Research Randomizer, Social Psychology Network, Wesleyan University, Middletown, Conn.). Perceived hunger and fullness at baseline and post-exercise were measured along with postexercise ad libitum food intake. Each treatment was performed in duplicate ( 6 trials total) with at least 2 days between sessions. Subjects were instructed to refrain from structured exercise and alcohol consumption the day before and the day of each testing session, and to consume a selfselected ad libitum meal approximately $3 \mathrm{~h}$ prior to each testing session.

\section{Exercise treatments}

The subjects arrived at the exercise testing facility between 1600 and 1800 hours, whereupon they emptied their bladders and then their body weights were recorded (Sartorius Combics IS, Data Weighing Systems, Elk Grove, Ill.). The AEx sessions consisted of a 35min bout of pedaling on a cycle ergometer (Ergomedic 828E) at 70\% heart rate reserve with 5 min given for warm up and cool down. The REx sessions consisted of 2 sets of 10 repetitions and a third set to voluntary fatigue (or 12 repetitions) at $70 \%$ of 1RM for chest press, double leg press, seated leg extension, seated leg curl, and seated row. One minute of rest was required between each set. During the CON session, the subjects were seated in the exercise room and were permitted to read, watch TV, or work on school assignments. Conditions in the exercise room remained constant $\left(\sim 21.3{ }^{\circ} \mathrm{C}, \sim 25 \%\right.$ humidity $)$ for each testing session. Additionally, each subject consumed $354 \mathrm{~mL}$ of water during each session. One to 5 subjects were tested on a given day.

\section{Perceived appetite}

Perceived hunger and fullness were assessed 60 min pre-testing, immediately postintervention ( $+45 \mathrm{~min}$ ), immediately before starting to eat (+75 min), and $30 \mathrm{~min}$ after starting the meal (+105 min). A 13-point numerical rating scale (arbitrary units) with the left 
anchor "1" of "not at all" and the right anchor "13" of "extremely" was utilized (DiMeglio and Mattes 2000). This type of assessment tool has been validated elsewhere (Green et al. 1996).

MEI

Thirty minutes after the AEx, REx, or CON session ended, each subject was seated at a table and served a large bowl of pasta salad cooled to $4{ }^{\circ} \mathrm{C}$. The subjects were asked to consume this meal within $30 \mathrm{~min}$, and were instructed to eat as much or as little as desired until feeling comfortably full. They were also provided with $237 \mathrm{~mL}$ of water which they were instructed to completely consume with the meal. The subjects were supervised, silent, and partitioned from each other while eating. The bowl with the pasta and the utensils were weighed before and after the meal to measure how much food was consumed. The subjects knew their food intake was being recorded. The pasta salad was made with Barilla Tri Color Rotini, Kraft Light Done Right Italian dressing, and Kraft shredded parmesan cheese. Each $400-\mathrm{g}$ serving of the pasta salad contained $610 \mathrm{kcal}(1 \mathrm{kcal}=4.184 \mathrm{~kJ})$ energy, $89 \mathrm{~g}$ carbohydrate, $23 \mathrm{~g}$ protein, and $19 \mathrm{~g}$ fat; subjects were given $\sim 3$ servings in the large bowl from which they served themselves onto a dinner-size plate.

\section{Energy expenditure and relative energy intake (REI)}

Metabolic equivalents (METs) were used to estimate the energy expenditure of exercise (EEEx) for AEx (ACSM 2006). A value of 8 METs was used to estimate energy expenditure per minute of AEx. The EEEx for REx was estimated using the following equation: (35 min $\times$ body weight $(\mathrm{kg}) \times 0.141) / 4.184)($ Campbell et al. 1994). These estimates of EEEx do not include the person's resting energy expenditure during the exercise. The EEEx of the control was considered to be zero. REI was estimated by subtracting the EEEx from the energy intake of the meal.

\section{Reproducibility and statistical analysis}

Each subject's data from the duplicate testing days for each treatment were analyzed for reproducibility using the intraclass correlation coefficient (ICC), the Pearson correlation $(r)$, and Bland-Altman plots. An ICC $\geq 0.8$ is considered to show good reproducibility, $0.7 \leq$ ICC $<0.8$ shows moderate reproducibility, and $0.6 \leq$ ICC $<0.7$ shows an acceptable level of reproducibility (Choi et al. 2000). The results from the duplicate testing days were averaged and these within-subject mean values were used to assess the treatment effects.

Repeated measures analysis of variance with a mixed model approach was used to assess the effects of treatment on food intake, and treatment and time on indices of appetite. Post hoc analysis was performed using Tukey's multiple comparison test to evaluate betweentreatment effects at each time point. Hunger data were transformed using power of 0.75 and fullness data were transformed using the natural logarithm to achieve a normal distribution. Inclusion of a subject's sex in the statistical model did not impact the primary findings (i.e., there were no sex-by-treatment, sex-by-time, or sex-by-treatment-by-time interactions). Because the study was not designed to evaluate the main effect of sex, the results are presented without statistically accounting for this independent variable. Statistical Analysis

Appl Physiol Nutr Metab. Author manuscript; available in PMC 2015 September 08. 
Software version 9.2 was used for all statistical analysis (SAS Institute Inc., Cary, N.C.). All values are reported as means \pm SD. Significant $p$ value is $\leq 0.05$.

\section{Results}

\section{Subject characteristics}

Subject characteristics are presented in Table 1.

\section{Exercise}

All 19 subjects successfully completed duplicate AEx, REx, and CON treatments. The subjects averaged $70.2 \% \pm 3.7 \%$ of their heart rate reserve for the 35 min of cycling during AEx, which equaled $80.7 \% \pm 2.5 \%$ of $V \dot{O}_{2 \text { max }}$. All subjects were able to fully complete the REx protocol at their prescribed resistances. The estimated EEEx of AEx and REx were 290 $\pm 7 \mathrm{kcal}$ and $80 \pm 2 \mathrm{kcal}$, respectively $(p<0.05)$.

\section{Appetite response}

Perceived hunger was reduced immediately following AEx (+45 min) compared with preexercise $(-60 \mathrm{~min})(p=0.03)$ (Fig. 1A). This was followed by an increase in postexercise hunger $(+75 \mathrm{~min})$ prior to the meal $(p<0.0001)$. In contrast, perceived hunger was not changed following REx (+45 min) compared with pre-exercise (-60 min), but increased prior to the meal (i.e., from +45 to $+75 \mathrm{~min})(p=0.0007)$. This REx response was not different than CON. Immediately postexercise (+45 min), hunger was lower for AEx vs. REx and CON (AEx vs. CON, $p<0.0001$; AEx vs. REx, $p=0.05$ ). Perceived hunger decreased following the ad libitum meal with no differences in response among treatments. Independent of treatment, fullness was lower at minute +75 vs. minute $-60(p=0.0004)$, and increased after the meal $(p<0.0001)$ (Fig. 1B).

\section{Acute food intake response}

Mean MEI of the pasta salad meal was $897 \pm 96 \mathrm{kcal}, 924 \pm 95 \mathrm{kcal}$, and $784 \pm 89 \mathrm{kcal}$ for the AEx, REx, and CON, respectively (Supplementary Fig. S1 ${ }^{2}$ ). Thus, REx and AEx led to a higher MEI compared with CON (REx vs. CON, $p=0.02$; AEx vs. CON, $p=0.04$ ). No difference in MEI was observed between AEx and REx. When adjusting for the EEEx, mean REI was found to be $607 \pm 94 \mathrm{kcal}, 844 \pm 91 \mathrm{kcal}$, and $784 \pm 89 \mathrm{kcal}$ for the AEx, REx, and CON sessions, respectively (Supplementary Fig. S1 ${ }^{2}$ ). Aerobic exercise led to lower REI compared with the REx and CON sessions (AEx vs. REx, $p<0.0001$; AEx vs. CON, $p=$ 0.003). No difference in REI was observed between REx and CON.

\section{Reproducibility}

MEI was highly associated within treatment for AEx, REx, and CON $(r=0.853 \mathrm{AEx}, r=$ $0.818 \mathrm{REx}, r=0.756 \mathrm{CON} ; p<0.0001$ ) (Fig. 2). The ICCs for AEx, REx, and CON all showed a good level of reproducibility (ICC $=0.899 \mathrm{AEx}, \mathrm{ICC}=0.894 \mathrm{REx}, \mathrm{ICC}=0.857$

\footnotetext{
${ }^{2}$ Supplementary data for this article are available on the journal Web site (http://apnm.nrc.ca).
} 
CON). The spread of the data points on the Bland-Altman plots were evenly above and below the mean, indicating that there was no systematic bias (Supplementary Fig. S2 ${ }^{2}$ )

\section{Discussion}

The primary goal of this project was to identify whether differences in postexercise appetite and food intake exist following resistance vs. aerobic exercise and sedentary states in young active adults. While a difference in hunger existed between REx and AEx immediately postexercise, this differential response was transient, and the predominant finding was that both modes of exercise increased MEI at the next eating occasion 30 min postexercise compared with remaining sedentary.

The finding that REx did not influence hunger immediately postintervention, compared with CON, contrasts with decreased hunger post-REx in young men who performed 3 sets of 10 exercises at $80 \%$ of 12RM (Broom et al. 2009). The young men in the Broom et al. (2009) study exercised about twice as long as our subjects (90 vs. $45 \mathrm{~min}$ ). Post-intervention fullness responses were not reported and ad libitum food intake was not assessed by Broom et al (2009). The current results indicate that REx increased MEI, with the magnitude of increase comparable with the AEx response ( 18 and 14\%, respectively). Additional research is needed to confirm this novel REx finding. The lack of a differential hunger response and no difference in REI between REx and CON suggests that REx does not induce anorexia of exercise.

The current finding that hunger was suppressed by AEx is consistent with some previous research and supports the concept of anorexia of exercise (Thompson et al. 1988; Kissileff et al. 1990; Verger et al. 1992; King et al. 1994; Imbeault et al. 1997; Westerterp-Plantenga et al. 1997; Lluch et al. 2000; Pomerleau et al. 2004; Maraki et al. 2005; Broom et al. 2009). The subsequent increase in hunger to control levels at $30 \mathrm{~min}$ post-AEx reinforces the transient nature of this appetitive response, which is generally reported to last no longer than 30 to 60 min (Verger et al. 1994; Blundell et al. 2003; Martins et al. 2008). In contrast to the differential hunger responses between AEx and CON, fullness was not influenced by AEx. The apparent lack of effect of AEx on fullness is consistent with results from young men who exercised on a treadmill for $30 \mathrm{~min}$ at $35 \%$ and $75 \% \mathrm{VO}_{2} \max$, compared with a noexercise control session (Imbeault et al. 1997).

The observation that MEI was 14\% higher after AEx was performed, compared with CON, is consistent with some previous research. A review of similar studies found 2 studies that reported decreased energy intake, 6 studies that reported an increased energy intake, and 14 studies that reported no change in energy intake (Blundell and King 1999). Based on the identification of 6 studies with no change in energy intake after exercise compared with control, 3 studies with an increase in energy intake, and 1 study with a decrease, another reviewer concluded that there is no anorexia of exercise (Martins et al. 2008). The differences in findings among studies and interpretations between reviewers could be due to a variety of factors, including timing of the meal, intensity of the exercise, and types of foods offered. A higher MEI postexercise suggests that dietary compensation occurred, but lower REI suggests the dietary compensation was incomplete.

Appl Physiol Nutr Metab. Author manuscript; available in PMC 2015 September 08. 
A lower REI and decreased hunger are typically interpreted as anorexia of exercise. Our finding that REI was lower following AEx, compared with CON, is consistent with other research (Thompson et al. 1988; Kissileff et al. 1990; King et al. 1994; Verger et al. 1994; Imbeault et al. 1997; Westerterp-Plantenga et al. 1997; Lluch et al. 2000; Pomerleau et al. 2004; Maraki et al. 2005), and underscores the importance of the EEEx component when interpreting the effects of exercise on energy intake. It would seem plausible to postulate that higher AEx-induced EEEx would result in a lower REI and greater negative energy balance. While the intensity of exercise used to achieve a given total EEEx does not seem to influence post-AEx food intake (Pomerleau et al. 2004), higher EEEx achieved by performing higher-intensity vs. lower-intensity AEx for the same time period reduced both MEI and REI (Kissileff et al. 1990). It is important to note that the MEI and REI described above only reflect the ingestive behavior responses at the next eating occasion posttreatment. There is a general understanding that daily ingestive behavior is not tightly coupled with changes in EEEx and that people who change their habitual patterns of exercise do not necessarily experience a concurrent change in energy intake over longer periods of time (McGowan et al. 1986; Blundell and King 1998).

\section{Reproducibility}

The present finding that ad libitum MEI was not different between the duplicate CON trials, and there was a good level of reproducibility with an ICC $=0.869$, which is consistent with other research (Arvaniti et al. 2000; Gregersen et al. 2008; Nair et al. 2009). The investigators for these studies (Arvaniti et al. 2000; Gregersen et al. 2008; Nair et al. 2009) specifically requested the participants to avoid structured or vigorous physical activity for 2 days prior to testing. The intraclass correlations were ICC $=0.97$ (Arvaniti et al. 2000) and ICC $=0.89$ (Nair et al. 2009) when subjects were provided buffet-style meals, and the Pearson correlations when subjects were tested with and without diet standardization the day before testing were $r=0.861$ and $r=0.654$, respectively (Gregersen et al. 2008). The present study is the first to assess the reproducibility of MEI following exercise. The ICC = 0.899 and 0.894 for AEx and REx, respectively, indicating comparable reproducibility with CON.

\section{Strengths and limitations}

This is one of the first studies to assess the subjects' food intake following both resistance and aerobic exercise. The good reproducibility from the duplicate trials among the 3 modes of treatment strengthens the results. The subjects in this study were recreational athletes who habitually performed both aerobic and resistance exercise; thus, our findings are not confounded by the novelty-acclimation to performing these exercise bouts. One of the main limitations of this study is the indirect assessment of the REI from estimations of METs values with aerobic exercise and equations to estimate resistance exercise energy expenditure. The equation to estimate the EE of REx was established using indirect calorimetry data from a group of men that included participants in their 20s (mean age, $37 \pm$ 16 years) (Campbell et al. 1994). The men performed comparable resistance exercises (mode, intensity, number of repetitions and sets) as the participants in the current study. Under or over-estimation of the EEEx would increase or decrease, respectively, the estimation of REI and the subsequent interpretation of an anorexia of exercise effect. 
We chose to have the subjects perform AEx and REx for the same length of time at recommended intensities to broadly mimic how many adults perform exercise. We considered having the subjects expend the same amount of energy during both modes of exercise, but this would have resulted in very high intensity REx (increasing risk of injury), adopting a circuit-style REx (which would have confounded the comparison with AEx), or decreasing the intensity of AEx (which would have compromised the use of AEx as a comparison group for the anorexia of exercise). Future research is needed to evaluate the effects of AEx vs. REx when the EEEx of the 2 modes of exercise are the same.

\section{Conclusion}

This study uniquely assessed the effects of resistance exercise on hunger and food intake compared with aerobic exercise and no-exercise control. The ad libitum test meal used to measure spontaneous energy intake was reproducible under sedentary and post aerobic and resistance exercise conditions. The description that aerobic exercise induces anorexia of exercise is not consistent with the increased meal energy intake, but reflects the transient decrease in hunger and lower relative energy intake attributable to the energy expenditure of exercise. The novel findings that resistance exercise did not influence appetite sensations and increased meal energy intake suggest the concept of anorexia of exercise does not apply to resistance exercise.

\section{Acknowledgments}

The research participants and the members of Prof. Campbell's research staff are sincerely thanked for their dedication and contributions to this research. Special acknowledgement goes to Jan Green, Trent Wisehart, Ted Harper, Christine Wilkerson, and Vanessa Kobza for their technical help with the project. WWC, HJL, and DJL originated the study, developed the protocol, and provided supervision of the research. DJL performed the testing, data collection, and data processing. EL performed statistical analyses and consulted on the final manuscript. DJL and WWC wrote the manuscript, and HJL provided technical editing. All co-authors contributed to data interpretation and finalization of the manuscript. The authors have no conflicts of interest. The study was supported by a NIH R01 AG021911 grant. Support for HJL was provided in part by an Ingestive Behavior Research Center postdoctoral fellowship from Purdue University.

\section{References}

American College of Sports Medicine. ACSM's guidelines for exercise testing and prescription. 7. Lippincott Williams \& Wilkins; Baltimore, Md: 2006.

Arvaniti K, Richard D, Tremblay A. Reproducibility of energy and macronutrient intake and related substrate oxidation rates in a buffet-type meal. Br J Nutr. 2000; 83(5):489-495. [PubMed: 10953673]

Ballard TP, Melby CL, Camus H, Cianciulli M, Pitts J, Schmidt S, Hickey MS. Effect of resistance exercise, with or without carbohydrate supplementation, on plasma ghrelin concentrations and postexercise hunger and food intake. Metabolism. 2009; 58(8):1191-1199.10.1016/j.metabol. 2009.03.018 [PubMed: 19497597]

Blundell JE, King NA. Effects of exercise on appetite control: loose coupling between energy expenditure and energy intake. Int J Obes Relat Metab Disord. 1998; 22(Suppl 2):S22-S29. [PubMed: 9778093]

Blundell JE, King NA. Physical activity and regulation of food intake: current evidence. Med Sci Sports Exerc. 1999; 31(Suppl 11):S573-S583. [PubMed: 10593531]

Blundell JE, Stubbs RJ, Hughes DA, Whybrow S, King NA. Cross talk between physical activity and appetite control: does physical activity stimulate appetite? Proc Nutr Soc. 2003; 62(3):651661.10.1079/PNS2003286 [PubMed: 14692601] 
Broom DR, Batterham RL, King JA, Stensel DJ. Influence of resistance and aerobic exercise on hunger, circulating levels of acylated ghrelin, and peptide YY in healthy males. Am J Physiol Regul Integr Comp Physiol. 2009; 296(1):R29-R35. [PubMed: 18987287]

Campbell WW, Crim MC, Young VR, Evans WJ. Increased energy requirements and changes in body composition with resistance training in older adults. Am J Clin Nutr. 1994; 60(2):167-175. [PubMed: 8030593]

Choi JS, Wexner SD, Nam YS, Mavrantonis C, Salum MR, Yamaguchi T, et al. Intraobserver and interobserver measurements of the anorectal angle and perineal descent in defecography. Dis Colon Rectum. 2000; 43(8):1121-1126.10.1007/BF02236560 [PubMed: 10950011]

DiMeglio DP, Mattes RD. Liquid versus solid carbohydrate: effects on food intake and body weight. Int J Obes Relat Metab Disord. 2000; 24(6):794-800.10.1038/sj.ijo.0801229 [PubMed: 10878689]

Green BG, Dalton P, Cowart B, Shaffer G, Rankin K, Higgins J. Evaluating the 'Labeled Magnitude Scale' for measuring sensations of taste and smell. Chem Senses. 1996; 21(3):323-334.10.1093/ chemse/21.3.323 [PubMed: 8670711]

Gregersen NT, Flint A, Bitz C, Blundell JE, Raben A, Astrup A. Reproducibility and power of ad libitum energy intake assessed by repeated single meals. Am J Clin Nutr. 2008; 87(5):1277-1281. [PubMed: 18469250]

Imbeault P, Saint-Pierre S, Alméras N, Tremblay A. Acute effects of exercise on energy intake and feeding behaviour. Br J Nutr. 1997; 77(4):511-521.10.1079/BJN19970053 [PubMed: 9155502]

King NA, Burley VJ, Blundell JE. Exercise-induced suppression of appetite: effects on food intake and implications for energy balance. Eur J Clin Nutr. 1994; 48(10):715-724. [PubMed: 7835326]

Kissileff HR, Pi-Sunyer FX, Segal K, Meltzer S, Foelsch PA. Acute effects of exercise on food intake in obese and nonobese women. Am J Clin Nutr. 1990; 52(2):240-245. [PubMed: 2375289]

Lluch A, King NA, Blundell JE. No energy compensation at the meal following exercise in dietary restrained and unrestrained women. Br J Nutr. 2000; 84(2):219-225. [PubMed: 11029973]

Maraki M, Tsofliou F, Pitsiladis YP, Malkova D, Mutrie N, Higgins S. Acute effects of a single exercise class on appetite, energy intake and mood. Is there a time of day effect? Appetite. 2005; 45(3):272-278.10.1016/j.appet.2005.07.005 [PubMed: 16157416]

Martins C, Morgan L, Truby H. A review of the effects of exercise on appetite regulation: an obesity perspective. Int J Obes (London). 2008; 32(9):1337-1347.10.1038/ijo.2008.98 [PubMed: 18607378]

McGowan CR, Epstein LH, Kupfer DJ, Bulik CM, Robertson RJ. The effect of exercise on nonrestricted caloric intake in male joggers. Appetite. 1986; 7(1):97-105. [PubMed: 3963802]

Nair NS, Brennan IM, Little TJ, Gentilcore D, Hausken T, Jones KL, et al. Reproducibility of energy intake, gastric emptying, blood glucose, plasma insulin and cholecystokinin responses in healthy young males. Br J Nutr. 2009; 101(7):1094-1102.10.1017/S0007114508042372 [PubMed: 18680633]

Pomerleau M, Imbeault P, Parker T, Doucet E. Effects of exercise intensity on food intake and appetite in women. Am J Clin Nutr. 2004; 80(5):1230-1236. [PubMed: 15531670]

Siri WE. The gross composition of the body. Adv Biol Med Phys. 1956; 4:239-280. [PubMed: 13354513]

Thompson DA, Wolfe LA, Eikelboom R. Acute effects of exercise intensity on appetite in young men. Med Sci Sports Exerc. 1988; 20(3):222-227.10.1249/00005768-198806000-00002 [PubMed: 3386499]

Verger P, Lanteaume MT, Louis-Sylvestre J. Human intake and choice of foods at intervals after exercise. Appetite. 1992; 18(2):93-99.10.1016/0195-6663(92)90186-A [PubMed: 1610165]

Verger P, Lanteaume MT, Louis-Sylvestre J. Free food choice after acute exercise in men. Appetite. 1994; 22(2):159-164.10.1006/appe.1994.1015 [PubMed: 8037440]

Westerterp-Plantenga MS, Verwegen CR, Ijedema MJ, Wijckmans NE, Saris WH. Acute effects of exercise or sauna on appetite in obese and nonobese men. Physiol Behav. 1997; 62(6):13451354.10.1016/S0031-9384(97)00353-3 [PubMed: 9383124] 

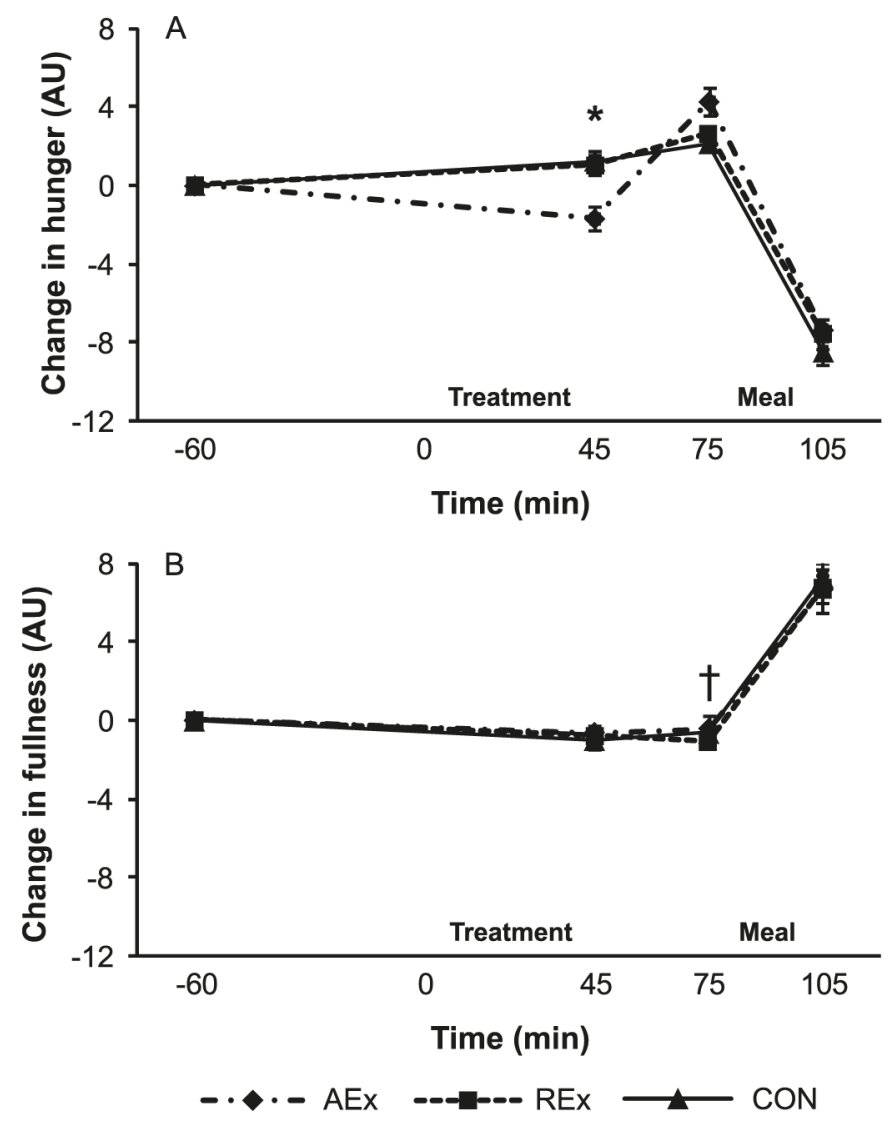

Fig. 1.

Hunger (A) and fullness (B) responses when young, physically fit adults performed aerobic exercise (AEx), resistance exercise (REx), or remained sedentary (control (CON)). *, AEx different than REx and CON at minute 45, $p=0.05$ and $p<0.0001$, respectively; $\dagger$, fullness lower at minute 75 vs. minute $-60(p=0.0004)$ and minute $105(p<0.0001)$ for all subjects combined, independent of treatment. 

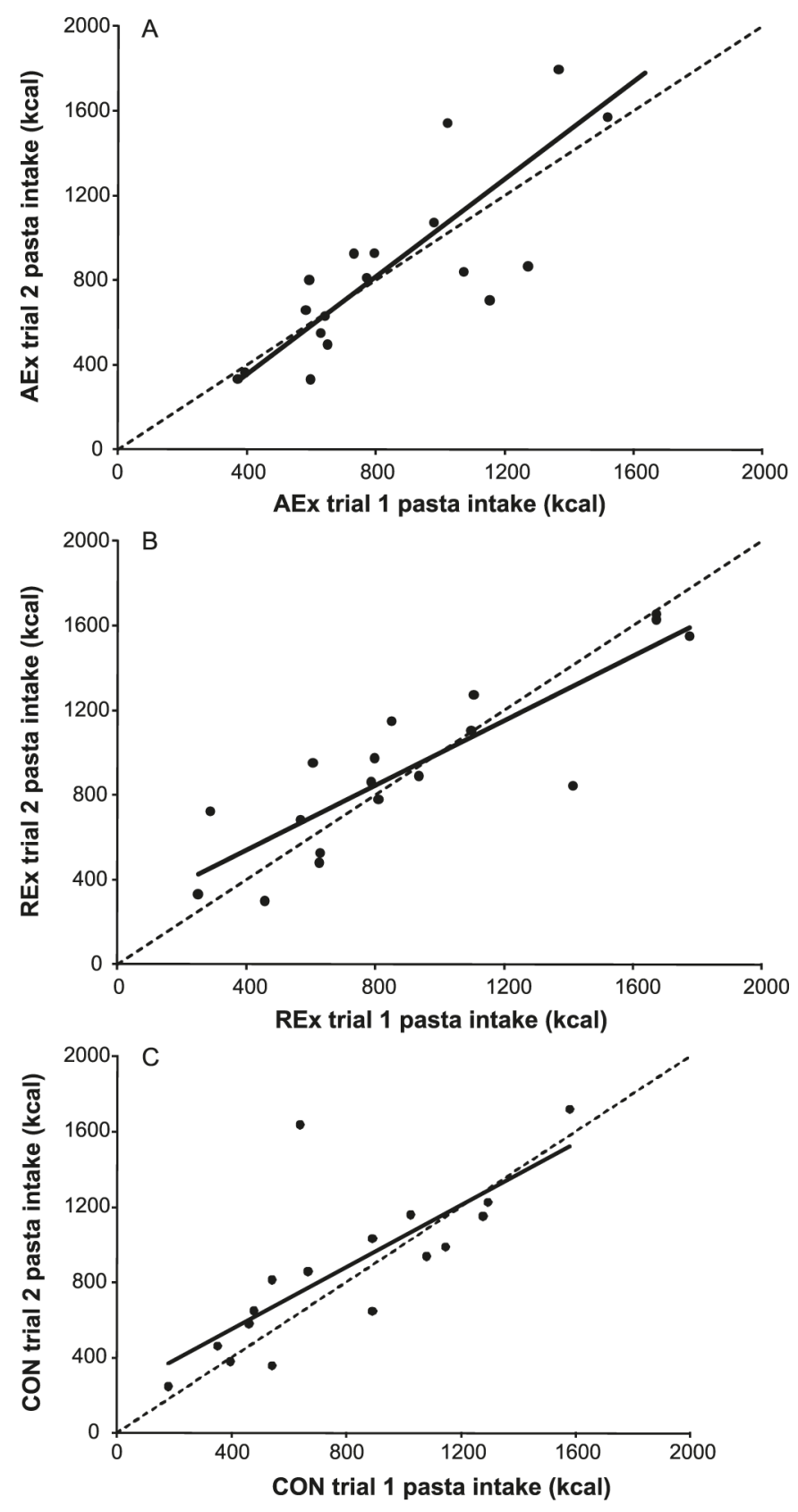

Fig. 2.

Ad libitum meal energy intake of pasta salad on duplicate days of testing 30 min after the subjects performed aerobic exercise (AEx, $r=0.853$; (A)), resistance exercise (REx, $r=$ 0.818 ; (B)), or no exercise control (CON, $r=0.773$; (C)). The dotted line is the identity line $(x=y)$. Each Pearson correlation $(r)$ was statistically significant, $p<0.0001 .1 \mathrm{kcal}=4.184$ $\mathrm{kJ}$. 


\section{Table 1}

Subject characteristics of 19 physically active young men and women.

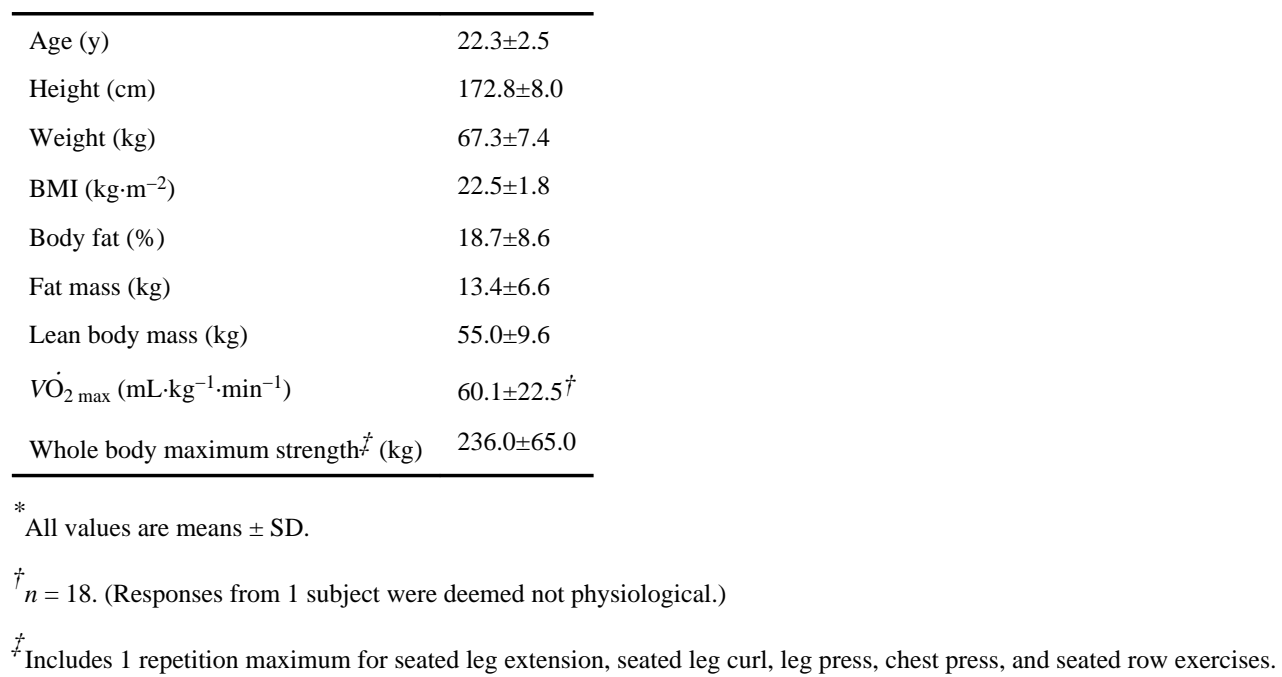

